Journal of Applied AnALysis

Vol. 9, No. 2 (2003), pp. 249-260

\title{
ON CONTINUOUS SELECTION PROBLEMS FOR MULTIVALUED MAPPINGS WITH THE LOCAL INTERSECTION PROPERTY IN HYPERCONVEX METRIC SPACES
}

\author{
X. WU, B. THOMPSON and X. YUAN \\ Received October 14, 2002 and, in revised form, April 22, 2003
}

\begin{abstract}
In the paper we present two continuous selection theorems in hyperconvex metric spaces and apply these to study fixed point and coincidence point problems as well as variational inequality problems in hyperconvex metric spaces.
\end{abstract}

\section{Introduction and preliminaries}

In 1956, Aronszajn and Panitchpakdi [1] first gave the notion of hyperconvex metric space, and then, Sine [16] and Soardi [17] proved independently that the fixed point property for nonexpansive mappings holds in bounded hyperconvex metric spaces. Since then, hyperconvex metric spaces has been widely studied and many interesting fixed point theorems for nonexpansive mappings has been established (for example, see [3, 5, 6, 7, 10, 11, 15] and

2000 Mathematics Subject Classification. 47H04, 47H10, 52A99, 54H25, 58E35.

Key words and phrases. Hyperconvex metric spaces, local intersection property, subadmissible set, acyclic set.

This work is supported in part by National Natural Science Foundation of China and Foundation of Australian Research Council and Natural Science Foundation of Chinese Yunnan Province.

ISSN 1425-6908 C Heldermann Verlag. 
others). The corresponding linear theory is well developed and associated with the names of Gleason, Goodner, Kelley and Nachbin. We refer readers to the reference [13] for more details. However, the nonlinear theory is still being developed. Recently, Khamsi, Kirk and Yañez [9] studied selection problems for multivalued mappings with bounded externally hyperconvex values.

In the present paper, our purpose is to study continuous selection problems for multivalued mappings with sub-admissible values. As applications of our new continuous selection theorems, we give the existence result for some fixed point and concidence point problems as well as for a generalized quasi-variational inequality problem and Fan's minimax inequality problem in hyperconvex metric spaces.

To begin with we explain the notion of a hyperconvex metric space and some related concepts. Hyperconvex metric spaces were introduced by Aronszajn and Panitchpakdi [1].

Definition 1.1. A metric space $(M, d)$ is called hyperconvex if for any collection of points $\left\{x_{\alpha}: \alpha \in I\right\}$ of $M$ and any collection of non-negative reals $\left\{r_{\alpha}: \alpha \in I\right\}$ such that

$$
d\left(x_{\alpha}, x_{\beta}\right) \leq r_{\alpha}+r_{\beta}
$$

for all $\alpha, \beta \in I$, then

$$
\bigcap_{\alpha \in I} \bar{B}\left(x_{\alpha}, r_{\alpha}\right) \neq \emptyset
$$

where $B(x, r)=\{y \in M: d(x, y)<r\}, \bar{B}(x, r)=\{y \in M: d(x, y) \leq r\}$.

Definition 1.2. Let $(M, d)$ be a metric space and $A \subset M$ a nonempty subset. Set

$$
\operatorname{co}(A)=\bigcap\{B: B \text { is a closed ball such that } A \subset B\}
$$

and

$$
A+r=\bigcup_{a \in A} \bar{B}(a, r), \forall r \geq 0 .
$$

If $A$ is an intersection of some closed balls, we will say $A$ is an admissible subset of $M . A$ is called sub-admissible if for each finite subset $D$ of $A, \operatorname{co}(D) \subset A$. A function $f: X \rightarrow \mathbb{R}$ is said to be metric quasi-convex (or metric quasi-concave) if for each $r \in \mathbb{R}$, the set $\{x \in X: f(x) \leq r\}$ (respectively, $\{x \in X: f(x) \geq r\}$ ) is sub-admissible.

Let $X$ be a topological space. We denote by $2^{X}$ the family of all subsets of $X$. If $A \subset X$ we shall denote by $\operatorname{cl}(A)$ the closure of $A$.

Definition 1.3. Let $X, Y$ be two topological spaces and $T: X \rightarrow 2^{Y}$ be a multivalued mapping. Then 
(1) $T$ is said to be transfer closed valued if for any $(x, y) \in X \times Y$ with $y \notin T(x)$, there exists a point $u \in X$ such that $y \notin \operatorname{cl}[T(u)]$;

(2) $T$ is said to be upper (respectively, lower) semicontinuous if for each $x \in X$ and each open set $V \subset Y$ with $T(x) \subset V$ (respectively, $T(x) \cap$ $V \neq \emptyset)$, there exists an open neighborhood $U$ of $x$ such that $T(z) \subset V$ (respectively, $T(z) \cap V \neq \emptyset$ ) for each $z \in U$;

(3) $T$ is said to have the local intersection property if for each $x \in X$ with $T(x) \neq \emptyset$ there exists an open neighborhood $N(x)$ of $x$ such that $\bigcap_{z \in N(x)} T(z) \neq \emptyset$.

Definition 1.4. Let $(M, d)$ be a metric space and $X \subset M$. A multivalued mapping $G: X \rightarrow 2^{M}$ is called a metric KKM-mapping if

$$
\operatorname{co}\left(\left\{x_{1}, \cdots, x_{n}\right\}\right) \subset \bigcup_{i=1}^{n} G\left(x_{i}\right)
$$

for any $x_{1}, \cdots, x_{n} \in X$.

A topological space is said to be acyclic if all of its reduced Čech homology groups over the rationals vanish. In particular, any contractible space is acyclic, and thus any convex or star-shaped set is acyclic.

\section{Continuous selection theorems}

Theorem 2.1. Let $X$ be a paracompact topological space, $(M, d)$ a hyperconvex metric space and $Y$ a nonempty sub-admissible subset of $M$. Let $T: X \rightarrow 2^{Y}$ be a multivalued mapping such that

(i) for each $x \in X, T(x)$ is a nonempty sub-admissible subset of $M$;

(ii) $T$ has the local intersection property.

Then $T$ has a continuous selection; that is, there exists a continuous mapping $f: X \rightarrow M$ such that $f(x) \in T(x)$ for all $x \in X$.

Proof. Since $(M, d)$ is a hyperconvex metric space, by the conclusion 1 of Proposition 1 in [8] there exists an index set $I$ and an isometric embedding from $M$ into $l^{\infty}(I)$. We will identify $M$ with the isometric embedding image set in $l^{\infty}(I)$. Since hyperconvexity is preserved by isometry, by the conclusion 4 of Proposition 1 in [8] there exists a nonexpansive retract $r: l^{\infty}(I) \rightarrow M$.

By (i) and (ii) we know that for each $x \in X$, there exists an open neighborhood $N(x)$ of $x$ such that the set $\bigcap_{z \in N(x)} T(z) \neq \emptyset$. Since $X$ is paracompact, there exists a locally finite open refinement $\mathcal{R}=\left\{U_{\alpha}: \alpha \in D\right\}$ of the family $\{N(x): x \in X\}$. Hence for each $\alpha \in D$, there exists a point $x_{\alpha} \in X$ 
such that $U_{\alpha} \subset N\left(x_{\alpha}\right)$. Note that $\bigcap_{z \in U_{\alpha}} T(z) \supset \bigcap_{z \in N\left(x_{\alpha}\right)} T(z) \neq \emptyset$, there exists a point $y_{\alpha} \in \bigcap_{z \in U_{\alpha}} T(z)$. Let $\left\{f_{\alpha}: \alpha \in D\right\}$ be a continuous partition of unity corresponding to the covering $\mathcal{R}$ of $X$. We can thus define a mapping $g: X \rightarrow l^{\infty}(I)$ by

$$
g(x)=\sum_{\alpha \in D} f_{\alpha}(x) y_{\alpha}, \quad \forall x \in X .
$$

Since $\mathcal{R}$ is a locally finite open covering of $X$ and $\left\{f_{\alpha}: \alpha \in D\right\}$ is a continuous partition of unity corresponding to $\mathcal{R}$, it follows that $g$ is a well-defined continuous mapping. Now let $f=r \circ g$. Then $f: X \rightarrow M$ is continuous. For each $x \in X$, let $\sigma(x, \mathcal{R})=\{U \in \mathcal{R}: x \in U\}$. Then $\sigma(x, \mathcal{R})$ is finite, and hence $\sigma(x, \mathcal{R})=\left\{U_{\alpha_{1}}, U_{\alpha_{2}}, \cdots, U_{\alpha_{n}}\right\}$ for some $\left\{\alpha_{1}, \alpha_{2}, \cdots, \alpha_{n}\right\} \subset D$. Therefore for each $x \in X$ we have

$$
\begin{aligned}
f(x) & =r[g(x)] \\
& =r\left[\sum_{k=1}^{n} f_{\alpha_{k}}(x) y_{\alpha_{k}}\right] \in r\left(\operatorname{conv}\left\{y_{\alpha_{1}}, \cdots, y_{\alpha_{n}}\right\}\right) \subset \operatorname{co}\left(\left\{y_{\alpha_{1}}, \cdots, y_{\alpha_{n}}\right\}\right),
\end{aligned}
$$

where $\operatorname{conv}\left\{y_{\alpha_{1}}, \cdots, y_{\alpha_{n}}\right\}$ is the convex hull of the $\left\{y_{\alpha_{1}}, \cdots, y_{\alpha_{n}}\right\}$ in $l^{\infty}(I)$. Since $\left\{y_{\alpha_{1}}, \cdots, y_{\alpha_{n}}\right\} \subset T(x)$ and $T(x)$ is a nonempty sub-admissible subset of $M$, it follows that $f(x) \in T(x)$. Therefore, $f$ is a continuous selection of $T$. This completes the proof.

From the proof of Theorem 2.1 it is clear that when $X$ is compact, we have the following result.

Theorem 2.2. Let $X$ be a compact topological space, $(M, d)$ a hyperconvex metric space, and $Y$ a nonempty sub-admissible subset of $M$. Let $T: X \rightarrow$ $2^{Y}$ be a multivalued mapping such that

(i) for each $x \in X, T(x)$ is a nonempty sub-admissible subset of $M$;

(ii) $T$ has the local intersection property.

Then $T$ has a continuous selection $f=r \circ g$, where $r: l^{\infty}(I) \rightarrow M$ is a nonexpansive retract, $I$ is some index set such that $M$ is isometrically embedded in $l^{\infty}(I)$ and $g: X \rightarrow l^{\infty}(I)$ is continuous such that $g(X)$ is contained in a polytope $P=\operatorname{conv}\left\{y_{1}, \cdots, y_{n}\right\}$ of $l^{\infty}(I),\left\{y_{1}, \cdots, y_{n}\right\} \subset Y$.

\section{Fixed point and coincidence point theorems}

As the first application of our continuous selection theorems we have the following fixed point theorem. 
Theorem 3.1. Let $(Y, d)$ be a hyperconvex metric space and $X$ be a nonempty compact admissible subset of $Y$. Suppose $T: X \rightarrow 2^{Y}$ is a multivalued mapping such that

(i) for each $x \in X, T(x)$ is a nonempty sub-admissible subset of $Y$;

(ii) $T$ has the local intersection property;

(iii) for each $x \in X$ with $x \notin T(x)$ and each $z \in T(x)$, there exists $\alpha \in(0,1)$ such that

$$
X \cap \bar{B}(x, \alpha d(x, z)) \cap \bar{B}(z,(1-\alpha) d(x, z)) \neq \emptyset .
$$

Then $T$ has a fixed point, that is, there exists a point $\bar{x} \in X$ such that $\bar{x} \in T(\bar{x})$.

Proof. By Theorem 2.1 there exists a continuous mapping $f: X \rightarrow Y$ such that $f(x) \in T(x)$ for all $x \in X$. For each $x \in X$, we define

$$
G(x)=\{y \in X: d(y, f(y)) \leq d(x, f(y))\} .
$$

Then $G: X \rightarrow 2^{Y}$ is a multivalued mapping with nonempty closed values. We claim that $G$ is a metric $K K M$ mapping. Otherwise, there exists a finite subset $A \subset X$ and a point $y \in \operatorname{co}(A)$ such that $y \notin \bigcup_{x \in A} G(x)$. Since $X$ is a sub-admissible subset of $Y$, it follows that $\operatorname{co}(A) \subset X$, and hence

$$
d(x, f(y))<d(y, f(y)), \quad \forall x \in A .
$$

Let $\varepsilon>0$ be such that

$$
d(x, f(y)) \leq d(y, f(y))-\varepsilon, \quad \forall x \in A .
$$

Then $A \subset X \cap \bar{B}(f(y), d(y, f(y))-\varepsilon)$. Hence $\operatorname{co}(A) \subset X \cap \bar{B}(f(y)$, $d(y, f(y))-\varepsilon)$. Consequently, $d(y, f(y)) \leq d(y, f(y))-\varepsilon$. This is a contradiction.

By the compactness of $X$ and Theorem 4 in [8], there exists a point $y_{0} \in \bigcap_{x \in X} G(x)$, that is,

$$
d\left(y_{0}, f\left(y_{0}\right)\right)=\inf _{x \in X} d\left(x, f\left(y_{0}\right)\right)
$$

We claim that $y_{0} \in T\left(y_{0}\right)$. Otherwise, since $f\left(y_{0}\right) \in T\left(y_{0}\right)$, by (iii) there exists $\alpha \in(0,1)$ such that

$$
X \cap \bar{B}\left(y_{0}, \alpha d\left(y_{0}, f\left(y_{0}\right)\right)\right) \cap \bar{B}\left(f\left(y_{0}\right),(1-\alpha) d\left(y_{0}, f\left(y_{0}\right)\right)\right) \neq \emptyset .
$$

Hence there exists a point $z \in X$ such that $d\left(y_{0}, z\right) \leq \alpha d\left(y_{0}, f\left(y_{0}\right)\right)$ and $d\left(f\left(y_{0}\right), z\right) \leq(1-\alpha) d\left(y_{0}, f\left(y_{0}\right)\right)$. Consequently, $d\left(f\left(y_{0}\right), z\right)=(1-\alpha) d\left(y_{0}\right.$, $\left.f\left(y_{0}\right)\right)$. Otherwise,

$$
d\left(y_{0}, f\left(y_{0}\right)\right)=\alpha d\left(y_{0}, f\left(y_{0}\right)\right)+(1-\alpha) d\left(y_{0}, f\left(y_{0}\right)\right)>d\left(y_{0}, z\right)+d\left(f\left(y_{0}\right), z\right),
$$

and hence

$$
d\left(y_{0}, f\left(y_{0}\right)\right) \leq d\left(y_{0}, z\right)+d\left(f\left(y_{0}\right), z\right)<d\left(y_{0}, f\left(y_{0}\right)\right) .
$$


This is a contradiction. Hence

$d\left(f\left(y_{0}\right), z\right)=(1-\alpha) d\left(y_{0}, f\left(y_{0}\right)\right)=(1-\alpha) \inf _{x \in X} d\left(x, f\left(y_{0}\right)\right) \leq(1-\alpha) d\left(z, f\left(y_{0}\right)\right)$.

We get a contradiction. This shows that $y_{0} \in T\left(y_{0}\right)$ and the proof is completed.

Corollary 3.2. Let $(Y, d)$ be a hyperconvex metric space and $X$ be a nonempty compact admissible subset of $Y$. Suppose that $T: X \rightarrow 2^{X}$ be a multivalued mapping such that

(i) for each $x \in X, T(x)$ is a nonempty sub-admissible subset in $Y$;

(ii) $T$ has the local intersection property.

Then $T$ has a fixed point.

Proof. For each $x \in X$ with $x \notin T(x)$ and each $y \in T(x), \operatorname{co}(\{x, y\}) \subset$ $X$ since $X$ is a nonempty sub-admissible subset of $Y$. Let $\operatorname{co}(\{x, y\})=$ $\bigcap_{j \in J} \bar{B}\left(x_{j}, r_{j}\right)$ and take any $\alpha \in(0,1)$. Since $d(x, y)=\alpha d(x, y)+$ $(1-\alpha) d(x, y), d\left(x, x_{j}\right) \leq r_{j} \leq r_{j}+\alpha d(x, y)$ and $d\left(y, x_{j}\right) \leq r_{j} \leq r_{j}+$ $(1-\alpha) d(x, y)$, by hyperconvexity of $Y, \operatorname{co}(\{x, y\}) \cap \bar{B}(x, \alpha d(x, y)) \cap \bar{B}(y,(1-$ $\alpha) d(x, y)) \neq \emptyset$, and hence $X \cap \bar{B}(x, \alpha d(x, y)) \cap \bar{B}(y,(1-\alpha) d(x, y)) \neq \emptyset$. This shows that condition (iii) in Theorem 3.1 is satisfied. Hence the conclusion follows from Theorem 3.1.

Remark . Corollary 3.2 contains Theorem 3.1 in [12] as a special case.

Theorem 3.3. Let $X$ be a compact topological space, $(M, d)$ a hyperconvex metric space and $Y$ a nonempty sub-admissible subset of $M$. Let $T: X \rightarrow 2^{Y}$ be a multivalued mapping with the local intersection property, let $F: Y \rightarrow 2^{X}$ be an upper semicontinuous multivalued mapping with closed acyclic values and let $T(x)$ be a nonempty sub-admissible subset of $M$ for each $x \in X$. Then there exist a point $x_{0} \in X$ and a point $y_{0} \in Y$ such that $x_{0} \in F\left(y_{0}\right)$ and $y_{0} \in T\left(x_{0}\right)$.

Proof. By Theorem 2.2, $T$ has a continuous selection $f=r \circ g$, where $r: l^{\infty}(I) \rightarrow M$ is a nonexpansive retract, $I$ is some index set such that $M$ is isometrically embedded in $l^{\infty}(I), g: X \rightarrow l^{\infty}(I)$ is continuous and, moreover, $g(X)$ is contained in a polytope $P=\operatorname{conv}\left\{y_{1}, \cdots, y_{n}\right\}$ of $l^{\infty}(I)$, for some subset $\left\{y_{1}, \cdots, y_{n}\right\} \subset Y$.

Let $S=\left.F \circ r\right|_{P}$. Then $S: P \rightarrow X$ is an upper semicontinuous multivalued mapping with compact acyclic values. By virtue of Lemma 2.1 in [14] there exists a point $z_{0} \in P$ such that $z_{0} \in g\left[S\left(z_{0}\right)\right]$. Hence there exists a point $x_{0} \in S\left(z_{0}\right)$ such that $z_{0}=g\left(x_{0}\right)$. Let $y_{0}=r\left(z_{0}\right)$. Then $y_{0}=r\left[g\left(x_{0}\right)\right]=$ $f\left(x_{0}\right) \in T\left(x_{0}\right)$ and $x_{0} \in F\left(y_{0}\right)$. This completes the proof. 


\section{Generalized quasi-variational inequalities}

As an application of our coincidence point theorems we have the following existence result for a generalized quasi-variational inequality problem.

Theorem 4.1. Let $M$ be a nonempty sub-admissible subset of a hyperconvex metric space $(Z, d)$, let $Y$ be a Hausdorff topological space and let $X \subset M$ be a nonempty compact admissible subset of $M$. Let $T: X \rightarrow 2^{Y}$ be an upper semicontinuous multivalued mapping with nonempty compact values. If an upper semicontinuous function $\phi: X \times Y \times M \rightarrow \mathbb{R}$ is such that

(i) for each $(x, y) \in X \times Y, \phi(x, y, z)$ is metric quasi-convex in $z$;

(ii) for each $(x, z) \in X \times M$, the set

$$
\{y \in T(x): \phi(x, y, z) \geq 0\}
$$

is acyclic;

(iii) for each $x \in X$, there exists a point $y \in T(x)$ such that $\phi(x, y, x) \geq 0$;

(iv) for each $x \in X$ and each $z \in M$ with $\sup _{y \in T(x)} \phi(x, y, z)<0$, there exists $\alpha \in(0,1)$ such that

$$
X \cap \bar{B}(x, \alpha d(x, z)) \cap \bar{B}(z,(1-\alpha) d(x, z)) \neq \emptyset,
$$

then there exists $\bar{x} \in X$ and $\bar{y} \in T(\bar{x})$ such that

$$
\phi(\bar{x}, \bar{y}, z) \geq 0
$$

for all $z \in M$.

Proof. We first prove that there exists a point $\bar{x} \in X$ such that

$$
\sup _{y \in T(\bar{x})} \phi(\bar{x}, y, z) \geq 0
$$

for all $z \in M$.

If the conclusion is false, then for each $x \in X$ there exists a point $v \in M$ such that

$$
\sup _{y \in T(x)} \phi(x, y, v)<0 .
$$

Let $S(x)=\left\{z \in M: \sup _{y \in T(x)} \phi(x, y, z)<0\right\}$. Then $S: X \rightarrow 2^{M}$ is a multivalued mapping with nonempty values and $S$ has no fixed point by (iii). For each $x \in X$, we claim that $S(x)$ is a sub-admissible subset of $M$. Indeed, for each finite subset $A$ of $S(x)$, there exists a real number $r<0$ such that

$$
\sup _{y \in T(x)} \phi(x, y, a) \leq r, \quad \forall a \in A
$$


Hence for each $z \in \operatorname{co}(A)$ and each $y \in T(x)$ we have $\phi(x, y, z) \leq r$ by (i). Consequently,

$$
\sup _{y \in T(x)} \phi(x, y, z) \leq r<0,
$$

that is, $z \in S(x)$. This shows that $S(x)$ is a sub-admissible subset of $M$.

Moreover, since $T: X \rightarrow 2^{Y}$ is an upper semicontinuous multivalued mapping with nonempty compact values and the function $\phi: X \times Y \times M \rightarrow \mathbb{R}$ is upper semicontinuous it follows by Proposition 21 in [2, p.119] that the function $\sup _{y \in T(x)} \phi(x, y, z)$ is upper semicontinuous in $x$, and hence $S$ has the local intersection property. Further, since condition (iv) implies that $S$ satisfies condition (iii) of Theorem 3.1, so by Theorem 3.1, the mapping $S$ has a fixed point. This is a contradiction since $S$ has no fixed points. Thus there exists a point $\bar{x} \in X$ such that

$$
\sup _{y \in T(\bar{x})} \phi(\bar{x}, y, z) \geq 0
$$

for all $z \in M$.

For each $z \in M$, let

$$
F(z)=\{y \in T(\bar{x}): \phi(\bar{x}, y, z) \geq 0\} .
$$

By the compactness of $T(\bar{x})$, the upper semicontinuity of $\phi$ and condition (ii) we know that $F: M \rightarrow 2^{T(\bar{x})}$ is an upper semicontinuous multivalued mapping with nonempty closed acyclic values. If the conclusion of Theorem 4.1 is false, the multivalued mapping $G: T(\bar{x}) \rightarrow 2^{M}$ defined by

$$
G(y)=\{z \in M: \phi(\bar{x}, y, z)<0\}
$$

has nonempty sub-admissible values and the local intersection property by (i) and the upper semicontinuity of $\phi$. Consequently, by Theorem 3.3 there exist a point $z_{0} \in M$ and a point $y_{0} \in T(\bar{x})$ such that $y_{0} \in F\left(z_{0}\right)$ and $z_{0} \in$ $G\left(y_{0}\right)$, that is, $\phi\left(\bar{x}, y_{0}, z_{0}\right) \geq 0$ and $\phi\left(\bar{x}, y_{0}, z_{0}\right)<0$. This is a contradiction. Therefore the conclusion of Theorem 4.1 is true.

If $X=M$ in Theorem 4.1, then condition (iv) is satisfied, automatically. Hence we have the following result.

Corollary 4.2. Let $(M, d)$ be a hyperconvex metric space, let $Y$ be a Hausdorff topological space and let $X$ be a nonempty compact admissible subset of $M$. Let $T: X \rightarrow 2^{Y}$ be an upper semicontinuous multivalued mapping with nonempty compact values. If an upper semicontinuous function $\phi: X \times Y \times X \rightarrow \mathbb{R}$ is such that

(i) for each $(x, y) \in X \times Y, \phi(x, y, z)$ is metric quasi-convex in $z$; 
(ii) for each $(x, z) \in X \times X$, the set

$$
\{y \in T(x): \phi(x, y, z) \geq 0\}
$$

is acyclic;

(iii) for each $x \in X$, there exists a point $y \in T(x)$ such that $\phi(x, y, x) \geq 0$, then there exists a point $\bar{x} \in X$ and a point $\bar{y} \in T(\bar{x})$ such that

$$
\phi(\bar{x}, \bar{y}, z) \geq 0
$$

for all $z \in X$.

Theorem 4.3. Let $M$ be a nonempty sub-admissible subset of a hyperconvex metric space $(Z, d)$, let $Y$ be a nonempty sub-admissible subset of a hyperconvex metric space and let $X \subset M$ be a nonempty compact admissible subset of $M$. Let $T: X \rightarrow 2^{Y}$ be a multivalued mapping with the local intersection property and nonempty sub-admissible values. If a function $\phi: X \times Y \times M \rightarrow \mathbb{R}$ is such that

(i) for each $(x, y) \in X \times Y, \phi(x, y, z)$ is metric quasi-convex in $z$;

(ii) for each $z \in M, \phi(x, y, z)$ is upper semicontinuous in $(x, y)$;

(iii) for each $x \in X$ and each $y \in T(x), \phi(x, y, x) \geq 0$;

(iv) for each $x \in X$ and each $z \in M$ with $\inf _{y \in T(x)} \phi(x, y, z)<0$, there exists $\alpha \in(0,1)$ such that

$$
X \cap \bar{B}(x, \alpha d(x, z)) \cap \bar{B}(z,(1-\alpha) d(x, z)) \neq \emptyset,
$$

then there exists a point $\bar{x} \in X$ and a point $\bar{y} \in T(\bar{x})$ such that

$$
\phi(\bar{x}, \bar{y}, z) \geq 0
$$

for all $z \in M$.

Proof. By Theorem 2.1 there exists a continuous mapping $f: X \rightarrow Y$ such that $f(x) \in T(x)$ for all $x \in X$.

If the conclusion is false, then for each $x \in X$ there exists a point $v \in M$ such that

$$
\phi(x, f(x), v)<0 .
$$

Let $S(x)=\{z \in M: \phi(x, f(x), z)<0\}$. Then $S: X \rightarrow 2^{M}$ is a multivalued mapping with nonempty sub-admissible values by (i). By (ii) we know that $S$ has the local intersection property. Since again condition (iv) implies that $S$ satisfies condition (iii) of Theorem 3.1, by Theorem $3.1 S$ has a fixed point $x^{*} \in X$, that is, $\phi\left(x^{*}, f\left(x^{*}\right), x^{*}\right)<0$. This contradicts (iii). Therefore, the conclusion of Theorem 4.1 is true. 


\section{Ky Fan's minimax inequality}

To facilitate the study of a Ky Fan type minimax inequality, we will use the following definition.

Definition 5.1. Let $X$ and $Y$ be two topological spaces and let $f: X \times Y \rightarrow$ $\mathbb{R}$ be a function. We call $f(x, y)$ transfer lower semicontinuous in $y$, if for each $r \in \mathbb{R}$ and each $(x, y) \in X \times Y$ with $f(x, y)>r$ there exists a point $x^{\prime} \in X$ and an open neighborhood $N(y)$ of $y$ such that $f\left(x^{\prime}, z\right)>r$ for all $z \in N(y)$.

Lemma 5.1. Let $(Y, d)$ be a hyperconvex metric space, $X$ be a nonempty compact admissible subset of $Y$ and $T: X \rightarrow 2^{Y}$ be a multivalued mapping. Suppose that the following conditions are satisfied:

(i) for each $y \in X, X \backslash T^{-1}(y)$ is a sub-admissible subset of $Y$;

(ii) for each $x \in X, x \in T(x)$;

(iii) $T$ is transfer closed valued.

Then $X \cap\left(\bigcap_{x \in X} T(x)\right) \neq \emptyset$.

Proof. For each $y \in X$, let $G(y)=X \backslash T^{-1}(y)$. Then $G: X \rightarrow 2^{X}$ is a multivalued mapping with sub-admissible values. For each $y \in X$ with $G(y) \neq \emptyset$, there exists a point $x \in G(y)$, that is, $y \notin T(x)$. By (iii) there exists a point $x_{0} \in X$ and an open neighborhood $N(y)$ of $y$ such that $N(y) \cap T\left(x_{0}\right)=\emptyset$. Consequently, for each $z \in X \cap N(y)$, we have $x_{0} \in G(z)$. This shows that $G$ has the local intersection property. Moreover, by (ii) we know that $G$ does not have any fixed points. Hence by Corollary 3.2 there exists a point $\bar{x} \in X$ such that $G(\bar{x})=\emptyset$, that is, $T^{-1}(\bar{x})=X$. Hence $\bar{x} \in \bigcap_{x \in X} T(x)$. This completes the proof.

Theorem 5.2. Let $(Y, d)$ be a hyperconvex metric space, $X$ be a nonempty compact admissible subset of $Y$ and $f: X \times Y \rightarrow \mathbb{R}$ be a function. Suppose that the following conditions are satisfied:

(i) for each $y \in Y, f(x, y)$ is metric quasi-concave in $x$;

(ii) $f(x, y)$ is transfer lower semicontinuous in $y$.

Then there exists a point $y_{0} \in X$ such that

$$
\sup _{x \in X} f\left(x, y_{0}\right)=\min _{y \in X} \sup _{x \in X} f(x, y) \leq \sup _{z \in X} f(z, z) .
$$

Proof. For each $x \in X$, let

$$
T(x)=\left\{y \in Y: f(x, y) \leq \sup _{z \in X} f(z, z)\right\} .
$$


Then $T: X \rightarrow 2^{Y}$ with $x \in T(x)$ for all $x \in X$. By (i) and (ii) we know that all conditions of Lemma 5.1 are satisfied. Hence by Lemma 5.1 there exists a point $y_{0} \in X$ such that $y_{0} \in \bigcap_{x \in X} T(x)$, that is, $f\left(x, y_{0}\right) \leq \sup _{z \in X} f(z, z)$ for all $x \in X$. Consequently,

$$
\sup _{x \in X} f\left(x, y_{0}\right)=\min _{y \in X} \sup _{x \in X} f(x, y) \leq \sup _{z \in X} f(z, z) .
$$

This completes the proof.

\section{References}

[1] Aronszajn, N., Panitchpakdi, P., Extension of uniformly continuous transformations and hyperconvex metric spaces, Pacific J. Math. 6 (1956), 405-439.

[2] Aubin, J. P., Ekeland, I., Applied Nonlinear Analysis, A Wiley-Interscience Publication, John Wiley \& Sons, Inc., New York, 1984.

[3] Baillon, J. B., Nonexpansive mapping and hyperconvex spaces, in "Fixed Point Theory and Its Applications", Contemp. Maths. 72, Amer. Math. Soc., Providence, RI, 1988, $11-19$.

[4] Fan, K., A generalization of Tychonoff's fixed point theorem, Math. Ann. 142 (1961), 305-310.

[5] Goebel, K., Kirk, W. A., Topics in Metric Fixed Point Theory, Cambridge University Press, Cambridge, 1990.

[6] Khamsi, M. A., Lin, M., Sine, R. C., On the fixed points of commuting nonexpansive maps in hyperconvex spaces, J. Math. Anal. Appl. 168 (1992), 372-380.

[7] Khamsi, M. A., Reich, S., Nonexpansive mappings and semigroups in hyperconvex spaces, Math. Japon. 35 (1990), 467-471.

[8] Khamsi, M. A., KKM and Ky Fan theorems in hyperconvex metric spaces, J. Math. Anal. Appl. 204 (1996), 298-306.

[9] Khamsi, M. A., Kirk, W. A., Yañez, C. M., Fixed point and selection theorems in hyperconvex metric spaces, Proc. Amer. Math. Soc. 128(11) (2000), 3275-3283.

[10] Kirk, W. A., Fixed point theory for nonexpansive mappings. II, in "Fixed Points and Nonexpansive Mappings", Contemp. Math. 18, Amer. Math. Soc., Providence, RI, 1983, 121-140.

[11] Kirk, W. A., Continuous mappings in compact hyperconvex metric spaces, Numer. Funct. Anal. Optim. 17(1996), 599-603.

[12] Kirk, W. A., Sims, B., Yuan, G. X.-Z., The Knaster-Kuratowski and Mazurkiewicz theory in hyperconvex metric spaces and some of its applications, Nonlinear Anal. 39 (2000), 611-627.

[13] Lacey, H. E., The Isometric Theory of Classical Banach Spaces, Springer, New York, 1974.

[14] Park, S., Bae, J. S., Kang, H. K., Geometric properties, minimax inequalities, and fixed point theorems on convex spaces, Proc. Amer. Math. Soc. 121(2) (1994), 429439.

[15] Sine, R. C., Hyperconvexity and approximate fixed points, Nonlinear Anal. 13 (1989), 863-869.

[16] Sine, R. C., On nonlinear contraction semigroups in sup-norm spaces, Nonlinear Anal. 3 (1979), 885-890. 
[17] Soardi, P. M., Existence of fixed points of nonexpansive mappings in certain Banach lattices, Proc. Amer. Math. Soc. 73 (1979), 25-29.

XIAN WU

DEPARTMENT OF MATHEMATICS

YUNNAN NORMAL UNIVERSITY

Kunming, YunNan 650092

P. R. China

BEVAN THOMPSON

DEPARTMENT OF MATHEMATICS

The University of QueEnsland

BRISBANE QLD 4072

Australia
XIANZHI YUAN

Department of Mathematics

The UnIVERsity of QUEENSLAND BRISBANE QLD 4072

Australia 